\title{
Functional and emotional outcomes after transient ischaemic attack: A 12-month prospective controlled cohort study.
}

Catherine M Sackley PhD

School of Public Health and Environmental Sciences, Kings College, London, UK Jonathan Mant MD

Primary Care Unit, Department of Public Health and Primary Care, Cambridge University, Cambridge, UK

Richard J McManus FRCGP

Department of Primary Care Health Sciences, University of Oxford, Oxford, UK Glyn Humphreys PhD RIP*

*Correspondence: Richard J McManus as above

Leslie Sharp

School of Public Health and Environmental Sciences, Kings College, London, UK Kathryn Mares PhD, Corresponding author

k.mares@uea.ac.uk, 01603593099

School of Health Sciences, University of East Anglia, Norwich, UK

George M Savva PhD

Quadram Institute Bioscience, Norwich Research Park, Norwich, UK Cover Title:

12-month outcomes after transient ischaemic attack 


\section{Tables:}

Table 1: Demographic, clinical characteristics and baseline outcome measures of FACE-TIA participants stratified by study diagnosis group.

Table 2:Difference in HADS-A, HADS-D and NEADL scores for each clinic group compared to controls, at baseline and 12 months.

Table 3:Change in anxiety, depression and disability between baseline and 12-month followup.

\section{Figures:}

Figure 1: Participant flow through the study.

Figure 2: Box plots showing baseline distribution of HADS-A, HADS-D and NEADL scores among controls and cases stratified by diagnosis group

Figure 3: Trajectories of group marginal means of anxiety, depression and function over 12 months from diagnosis in control, TIA and minor stroke groups

\section{Key words:}

Ischemic Attack, Transient; functional outcome; emotional outcome

\section{Subject Terms:}

Stroke; Epidemiology; Lifestyle and Prevention

\section{Word count:}




\section{Abstract}

\section{Background}

Symptoms of transient ischaemic attack (TIA) are believed to fully resolve within 24 hours of onset. Emerging evidence suggests that there may be prolonged functional and psychological impact, although studies have not been able to robustly identify whether these are the effect of TIA or changes usually associated with ageing. We describe trajectories of disability and risk of anxiety and depression among patients seen at TIA clinics over twelve months, compared to healthy controls.

\section{Methods}

Thirty TIA clinics across England participated. 1320 participants were included: 373 diagnosed with TIA, 186 with minor stroke, 310 with 'possible TIA', 213 with another condition mimicking a TIA and 238 controls recruited from primary care providers. Participants completed questionnaires after diagnosis then after three, six and twelve months. Outcomes were the Nottingham Extended Activities of Daily Living scale, and the Hospital Anxiety and Depression Scale. Mixed effects regression was used to estimate group differences and trajectories.

\section{Results}

At baseline, confirmed TIA patients scored 1.31 (s.e=0.28; $\mathrm{p}<0.001)$ HADS-Anxiety points, 0.51 HADS-Depression points ( $\mathrm{s} . \mathrm{e}=0.26 ; \mathrm{p}=0.056)$ and 2.6 NEADL points $(\mathrm{s} . \mathrm{e}=1.1 ; \mathrm{p}=0.020)$ worse than controls. At 12 months, the deficits were 0.78 (s.e $=0.30 ; p=0.008), 0.97$ (s.e $=0.23$; $\mathrm{p}<0.001)$ and $0.96(\mathrm{~s} . \mathrm{e}=0.92 ; \mathrm{p}=0.294)$ respectively. Differences among patients diagnosed with minor stroke were like or worse than TIA patients.

\section{Conclusions}


FACE TIA

TIA clinic patients may have functional and emotional impairments compared to the general population irrespective of final diagnosis. The presence of emotional symptoms or risk of developing anxiety or depression did not always fully recover and may increase. 
FACE TIA

\section{Introduction}

Transient Ischemic Attack (TIA) are brief episodes of neurological dysfunction resulting from focal cerebral ischaemia not associated with permanent cerebral infarction ${ }^{1}$. An estimate for the incidence of first-ever TIA in the UK is 50 people/100,000 population/year ${ }^{2}$. Approximately $11-14 \%$ will have a stroke within the first week following TIA, referral to a TIA clinic can reduce the relative risk by $80 \%^{3}$. There were approximately 150,000 new referrals to TIA clinics in England in $2005^{4}$.

Management and most existing research on TIA focuses on prescription of prophylactic medications and prevention of cardiovascular episodes or death ${ }^{5}$.It was assumed that patients did not experience long term sequelae related to TIA. However uncontrolled studies or those relying on routinely collected primary care data suggest that there are residual psychological and functional impairments ${ }^{6,7}$ that are important to patients and families ${ }^{8}$. These have however not been able to determine whether any residual changes were because of a healthy ageing process. We are unaware of any controlled study using primary data collection that has investigated the long-term effects of functional, emotional and cognitive outcomes in the first year following referral to TIA clinics in England with suspected TIA.

\section{Aims}

We undertook a controlled prospective observational study (FACE-TIA) of patients attending TIA clinics to understand how mood, cognitive and functional outcomes differ from the general population in the year following clinic attendance. We report their baseline deficits and subsequent trajectories of functional and emotional outcomes over 12 months, stratified by final clinic diagnosis and compared to a control sample of patients. Cognitive outcomes were measured with the Birmingham Cognitive Screen ${ }^{9}$. Measurement was discontinued and is not reported here because there was insufficient data to be able to make any inferences about the population sampled. 
FACE TIA

\section{Methods}

\section{Design}

A controlled prospective observational study including participants identified from TIA clinics and matched controls.

\section{Setting}

Thirty TIA clinics across four regions of England were identified following a call for expressions of interest via the National Stroke Research Network. Controls were recruited by the Primary Care Research Network (PCRN) from General Practices within the same geographical areas as the TIA clinics.

\section{Population}

The target population for FACE-TIA was the UK population without previous stroke or TIA, attending a TIA clinic. Patients attending from September 2010 to December 2012 were eligible for inclusion in the study if their appointment was for a new incident, they attended and received a final diagnosis within 14 days of their event, had no previous history of stroke or TIA and were deemed by a consultant stroke physician or neurologist to be able to selfcomplete postal questionnaires (no known cognitive impairment that would prohibit selfcompletion of questionnaires).

Following informed consent participants were assigned a study diagnosis by the consultant, (attending) stroke physician or neurologist at the TIA clinic..

(i) Confirmed TIA: a transient episode of neurological dysfunction caused by focal brain, spinal cord, or retinal ischemia, without acute infarction ${ }^{1}$.

(ii) Minor stroke: prolonged deficits suggestive of focal or global cerebral, spinal, or retinal dysfunction caused by central nervous system infarction ${ }^{1}$, not requiring hospital admission. 
FACE TIA

(iii) TIA mimic: another condition mimicking the symptoms of TIA (e.g. migraine, seizure and sepsis ${ }^{10}$.

(iv) Possible TIA: Clinical symptoms suggestive of TIA but insufficient evidence to make a clinical diagnosis of either TIA, stroke or TIA mimic.

Controls were recruited from primary care following identification from practice patient registers with the help of the Primary Care Research Network (PCRN). Practices within the same geographical areas as the TIA clinics were chosen based on their postcode meta-data, specifically urban-rural indicators and indices of multiple deprivation. Participating practices provided the study team with an anonymous database containing year of birth, gender and a unique ID for each of their patients from which potential controls without history of stroke or TIA were selected. To provide balance across key covariates, controls were recruited to match the demographic characteristics of confirmed TIA cases.

Participants were included from the time of recruitment to the first of withdrawal, death, loss to follow-up or new stroke.

\section{Data collection and analysis}

Self-completion questionnaires were given to participants or sent by mail on recruitment. Follow-up questionnaires were sent by mail at three, six and twelve months after the baseline. Data were collated anonymously and all analyses were carried out using Stata version 14.0. Mixed effects multilevel regression was used to model continuous outcome measures across time within individuals. Sociodemographic (age, sex, educational attainment and area-level deprivation) and number of chronic diseases and smoking status were included as covariates. Statistical significance corresponds to $\mathrm{p}<0.05$ for each comparison, but a second critical threshold of $p<0.0042$ is also shown calculated using the method of Benjamini and Hochberg ${ }^{11}$ to correct for the large number of hypotheses being tested. The interaction of time (as a categorical variable) by group was included in each model to estimate the 


\section{FACE TIA}

trajectories of each outcome in each group. Weighted prevalence of case-level anxiety and depression was estimated at each time point.

As subgroup analyses, we explored the effects on each primary outcome at baseline stratified by age group and by sex, and tested age and sex specific effects by adding the interactions with age or sex as appropriate to each mixed model.

\section{Measures}

Functional ability was measured using the Nottingham Extended Activities of Daily Living (NEADL) scale ${ }^{11}$. NEADL includes 22 items that measure independence in everyday activities and societal participation in four domains: mobility, 'kitchen' related activities, other 'domestic' activities and leisure activities. Each activity is scored as 0 (not done), 1 (done with help), 2 (on my own with difficulty) or 3 (on my own). The maximum score is 66 , higher scores reflect greater independence.

Emotional outcomes were measured using the Hospital Anxiety and Depression Scale (HADS), a screening tool for anxiety (HADS-A), and depressive symptoms (HADS-D) that is not dependent on physical symptoms ${ }^{13}$. HADS includes seven depression (HADS-D) and seven anxiety items (HADS-A), each scored 0-3. HADS is scored out of 42 or 21 for each subscale with higher scores indicating worse symptoms; 0.5 represents a moderate effect size corresponding to a minimally important difference ${ }^{14}$.

Data also collected at each assessment included self-reported diagnoses of other health conditions, physical activity, smoking and alcohol use.

\section{Ethical approval}

The study was approved by NRES Committee West Midlands - Solihull:10/H1206/36. 
FACE TIA

\section{Results}

\section{Characteristics of the sample}

Of 1,698 participants recruited, 1287 (76\%) were included at baseline (figure 1). 232 (18\%) controls, $364(28 \%)$ with confirmed TIA, 183 (14\%) with minor stroke, 297 (23\%) with a 'possible' TIA and $211(16 \%)$ with TIA mimic. Baseline questionnaires were sent within a median of 4 (IQR 2-8) days from the index event (Table 1). Participants were balanced with respect to the Index of Multiple Deprivation (IMD), but controls were more likely to have completed a degree $(24 \%)$ than TIA cases $(14 \%)$ or other groups. $85 \%$ of participants had complete covariate and outcome data at baseline, so complete case analyses was used throughout. $75 \%$ of participants completed 12 -month follow-up; $91 \%$ of controls returned the 12 -month questionnaire compared to $69 \%$ of TIA, $74 \%$ of stroke, $64 \%$ of possible TIA and $66 \%$ of 'TIA mimic' cases.

\section{Functional and emotional outcome}

Figure 2 shows the distributions of HADS and NEADL across groups and table 2 shows the baseline means of each outcome with standard deviation, suggesting a considerable degree of overlap but significantly worse average scores for clinic attendees than controls irrespective of final diagnosis.

\section{Anxiety}

At baseline, all clinic referral groups had significantly worse anxiety scores than controls (table 2; figure 3). There was a statistically significant improvement in anxiety among the TIA, possible TIA and TIA mimic groups between baseline and 12 months, but not for controls or patients diagnosed with stroke (table 3).

Depression 


\section{FACE TIA}

HADS-D scores were significantly worse among stroke and possible TIA groups compared to controls at baseline, but not for people with TIA or TIA mimic (table 2; figure 3).

Depression became worse in the TIA group, becoming significantly worse than in controls at 12 months (table 2; figure 3). Depression remained worse than controls among possible TIA and stroke patients at 12 months (table 2). Although improvements in anxiety were statistically significant after correcting for multiple testing (table 3) none of the changes in depression scores within groups were as statistically significant and directions of change were mixed across groups.

$N E A D L$

NEADL scores were lower among clinical groups than controls at baseline, although this was not statistically significant for TIA mimic group. NEADL scores improved in the TIA and stroke group over the 12 months of follow-up (table 3).

\section{Subgroup analysis}

There was no interaction between sex and diagnosis group for any baseline outcome (not shown). There were significant interactions between age and diagnosis group for emotional outcomes (supplemental table VI). We found that those under 70 with TIA, stroke and possible TIA had clinically and statistically significant increased depression and anxiety scores compared to control.

\section{Sensitivity analysis}

The pattern of results was not substantially altered when transformations to remove skew were applied to primary outcomes before estimating regression models (supplemental tables VII, VIII, and supplemental figure I).

\section{Discussion}




\section{FACE TIA}

We demonstrated that people attending TIA clinics have functional and emotional deficits when compared to the general population, particularly among people aged under 70 years. TIA or other diagnoses was not confirmed by a standardised protocol, but by the clinicians at each clinic and given that there is no diagnostic test for TIA, there may be overlap between people labelled as 'TIA', 'possible TIA' and 'mimic'. The study is of high clinical relevance for those people attending TIA clinics in the UK Neuroimaging studies of people with TIA have indicated that despite the potential for restoration of clinical symptoms evidence of brain damage is present on MRI ${ }^{1}$. Such damage may explain the presence of symptoms in the longer term but the lack of a control group in previous studies to FACE-TIA has not been able to robustly support this.

Patterns of deficits among those referred to TIA clinic but finally diagnosed with possible TIA or another condition mimicking a TIA were typically more like those diagnosed with TIA or stroke than to the healthy population. Degree level education was more prevalent in our control group than the diagnostic groups. This is unsurprising considering the reported positive relationship between education and health and was controlled for in regression analyses ${ }^{15}$. Anxiety was elevated in clinic attendees who did not have a confirmed TIA or stroke compared to controls, but the difference was not significant at 12 months. This most likely reflects the uncertainty caused by referral or due to other morbidity, but could also indicate a lower threshold for referral in anxious patients.

Despite improvements over the period of the study people diagnosed with TIA, possible TIA or stroke remained more likely to show symptoms of anxiety and less able to carry out activities of daily living than the control group at 12 months.

Symptoms of depression scores were worse than controls for TIA, possible TIA and stroke patients at 12 months and did not improve for the TIA mimic group. 


\section{FACE TIA}

Despite patient reported concerns ${ }^{8}$, little follow up data has been published describing anxiety after TIA. Our systematic review ${ }^{6}$ identified only the pilot study of FACE-TIA ${ }^{15}$ and data from an uncontrolled cohort of 49 non-severe stroke survivors at 12 months post event ${ }^{16}$. Since our review was conducted a cross sectional survey of 1247 patients from Glasgow, UK, estimated the prevalence of anxiety following TIA using a HADS cut-off of 11 to be $14 \%$, identical to our finding but did not report longitudinal data ${ }^{17}$.

More data are available on depression after TIA and minor stroke: between $8-42 \%$ experience depression within the first year post event, but again there are methodological shortcomings in many studies and time post-event is not recorded or is not consistent for the largest studies $^{6}$. In the cross-sectional survey discussed above, the population prevalence of probable depression (based on HADS-D score of 11 or more) after TIA was $9 \%$, higher than our estimated prevalence of $4.3 \%(95 \% \mathrm{CI}=2.3-7.8)$, possibly reflecting differences in geographic location between studies ${ }^{17}$.

Anxiety and depression were elevated in clinic attendees who did not have a confirmed TIA or stroke compared to controls, perhaps associated with the uncertainty caused by referral or due to other morbidity.

A deterioration in functional ability may contribute to the loss of self-confidence and feelings of insecurity in carrying out normal functions reported by patients following a TIA ${ }^{18}$.

The worsening depression in TIA group could reflect a vascular cause of depression ${ }^{19}$. An analysis of participants from the Whitehall study showed that depressive symptoms were linked to incident stroke risk and may reflect deteriorating cerebrovascular health ${ }^{20}$. The 'possible TIA' group had higher depression and anxiety scores than the TIA group and so emotional support may be needed for TIA referrals irrespective of final diagnosis. 


\section{FACE TIA}

This is the first prospective controlled study of people attending a TIA clinic compared to controls. We did not record potential confounders such as therapy received by participants during the follow up period however, the findings suggest that there are sufficient adverse psychological and functional consequences of TIA, minor stroke and other conditions referred to a TIA clinic to merit further attention - and intervention, both in terms of research and clinical rehabilitation. 
FACE TIA

\section{Acknowledgements}

We acknowledge the help of the stroke and primary care research networks, Sheridan

Bevan and members of the Institute of Applied Health Research at the University of

Birmingham. Thanks, go to NHS staff, patients and their families.

Professor Glyn Humphries died suddenly on $14^{\text {th }}$ January 2016 and was an integral member of the FACE-TIA team.

\section{Declaration of conflicting interests}

The Authors declare no conflict of interest.

\section{Funding}

This work was supported by the Stroke Association TSA 2011/09. 


\section{References}

1. Easton JD, Saver JL, Albers GW, et al. Definition and evaluation of transient ischemic attack. Stroke 2009; 40: 2276-2293.

2. Rothwell PM, Coull AJ, Silver LE, et al. Population-based study of event-rate, incidence, case fatality, and mortality for all acute vascular events in all arterial territories (Oxford Vascular Study). Lancet 2005; 366: 1773-1783. Research Support, Non-U.S. Gov't $2005 / 11 / 22$.

3. Rothwell PM, Giles MF, Chandratheva A, et al. Effect of urgent treatment of transient ischaemic attack and minor stroke on early recurrent stroke (EXPRESS study): a prospective population-based sequential comparison. Lancet 2007; 370: 1432-1442.

4. Giles MF and Rothwell PM. Substantial underestimation of the need for outpatient services for TIA and minor stroke. Age and Ageing 2007; 36: 676-680.

5. Cameron AC, Dawson J, Quinn TJ, et al. Long-term outcome following attendance at a transient ischemic attack clinic. Int J Stroke 2011; 6: 306-311.

6. Moran GM, Fletcher B, Feltham MG, et al. Fatigue, psychological and cognitive impairment following transient ischaemic attack and minor stroke: a systematic review. Eur $J$ Neurol 2014; 21: 1258-1267.

7. Turner GM, Calvert M, Feltham MG, et al. Ongoing impairments following transient ischaemic attack: retrospective cohort study. Eur J Neurol 2016; 23: 1642-1650.

8. Spurgeon L, Humphreys G, James G, et al. A Q-Methodology Study of Patients' Subjective Experiences of TIA. Stroke Res Treat 2012;

9. Humphreys G, Bickerton, W.-L., et al. BCoS Cognition Screen. Hove, England: Psychology Press., 2012.

10. Hand PJ, Kwan J, Lindley RI, et al. Distinguishing between stroke and mimic at the bedside: the brain attack study. Stroke 2006; 37: 769-775.

11. Benjamini Y, Yekutieli D. The control of the false discovery rate in multiple testing under dependency. Ann Stat. 2001; 29: 1165-88

11. Nouri FM and Lincoln NB. An extended activities of daily living scale for stroke patients. Clinical Rehabilitation 1987; 1: 301-305.

12. Zigmond AS and Snaith RP. The hospital anxiety and depression scale. Acta

Psychiatr Scand 1983; 67: 361-370.

13. Norman G, Sloan J and Wyrwich W. Interpretation of health-related quality of life: the remarkable universality of half a standard deviation. Med Care; 41: 5: 582-592

14. Link BG, Phelan J. Social conditions as fundamental causes of disease. J Health Soc Behav 1995; 36:80-9415.

15. Brittle N. "Functional, cognitive and emotional outcomes after transient ischaemic attack: a systematic review and controlled cohort study". Birmingham, 2012.

16. Bruggimann L, Annoni J, Staub F, et al. Chronic posttraumatic stress symptoms after nonsevere stroke. Neurology 2006; 66: 513-516.

17. Duncan PW, Samsa GP, Weinberger M, et al. Health status of individuals with mild stroke. Stroke 1997; 28: 740-745.

18. Spurgeon L, James G and Sackley C. Subjective experiences of transient ischaemic attack: a repertory grid approach. Disabil Rehabil 2013; 35: 2205-2212.

19. Thomas AJ, Kalaria RN and O'Brien JT. Depression and vascular disease: what is the relationship? J Affect Disord 2004; 79: 81-95.

20. Brunner EJ, Shipley MJ, Britton AR, et al. Depressive disorder, coronary heart disease, and stroke: dose-response and reverse causation effects in the Whitehall II cohort study. Eur J Prev Cardiol 2014; 21: 340-346. 


\section{Figures and tables for FACE main paper}

Figure 1: Participant flow through the study.

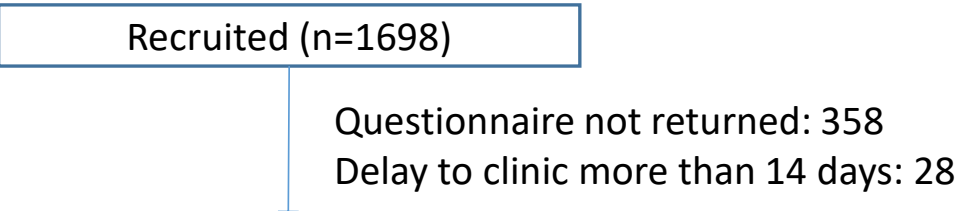

Baseline Questionnaire Returned ( $n=1312)$

Previous stroke reported in non-stroke group: 18

Previous TIA reported in control group: 6

Unknown diagnosis: 1

Included in Baseline Analysis ( $n=1287)$

Loss to follow-up: 168

Incident stroke in non-stroke group: 18

Incident TIA in control: 0

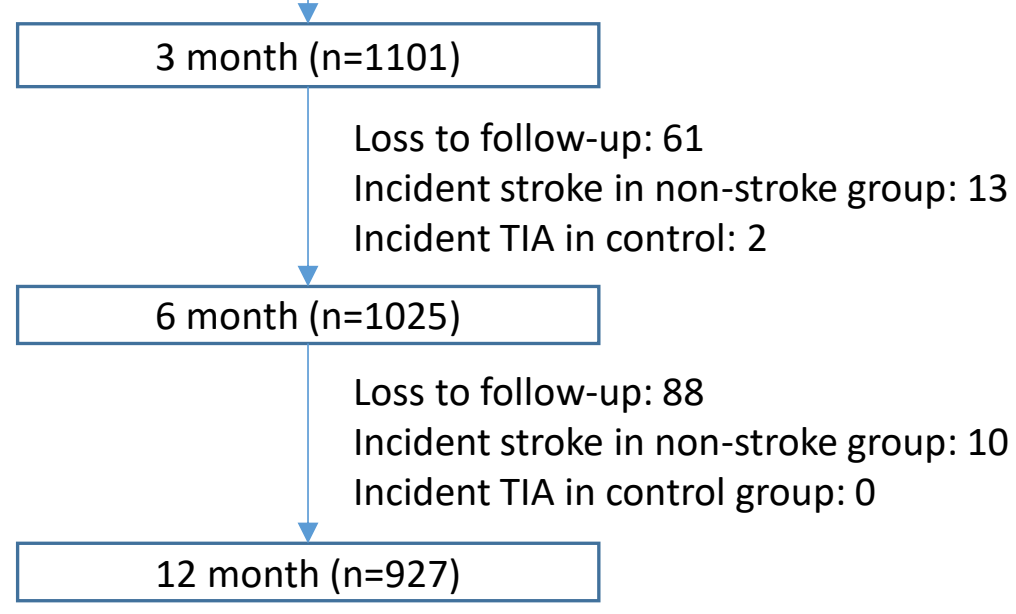


FACE TIA

Figure 2:

Box plots showing baseline distribution of HADS-A (panel A), HADS-D (panel B) and

NEADL (panel C) scores among controls and cases stratified by diagnosis group
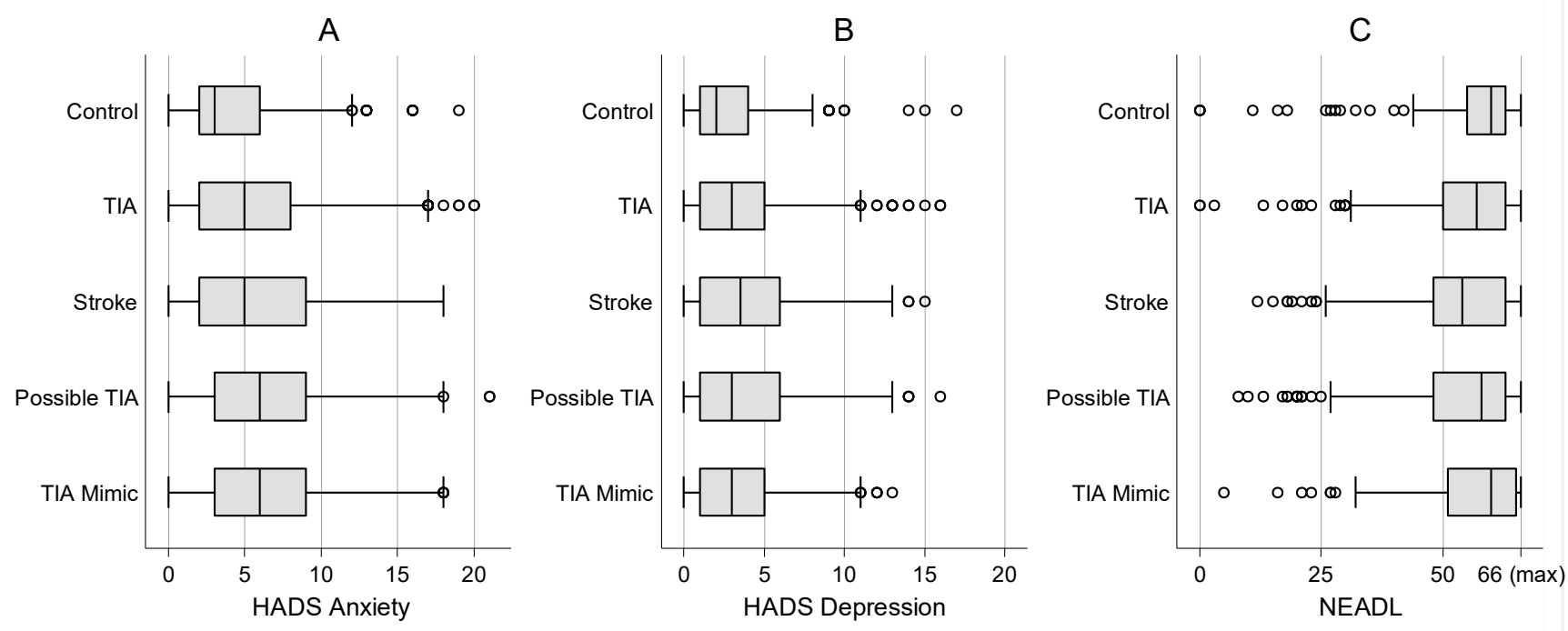
FACE TIA

Figure 3:

Trajectories of group marginal means of anxiety (panel A) depression (panel B) and function (panel C) over 12 months from diagnosis in control (hollow circles), TIA (filled circles) and minor stroke (no marker) groups. Error bars represent 95\% confidence intervals. Estimates are standardised to sample means of baseline covariates. Numerical values for primary outcomes across all diagnosis groups (supplemental tables III-V), and a figure showing the trajectories among the 'possible TIA' and 'TIA mimic' groups compared to controls (supplemental figure III) are supplied in the supplementary appendix.
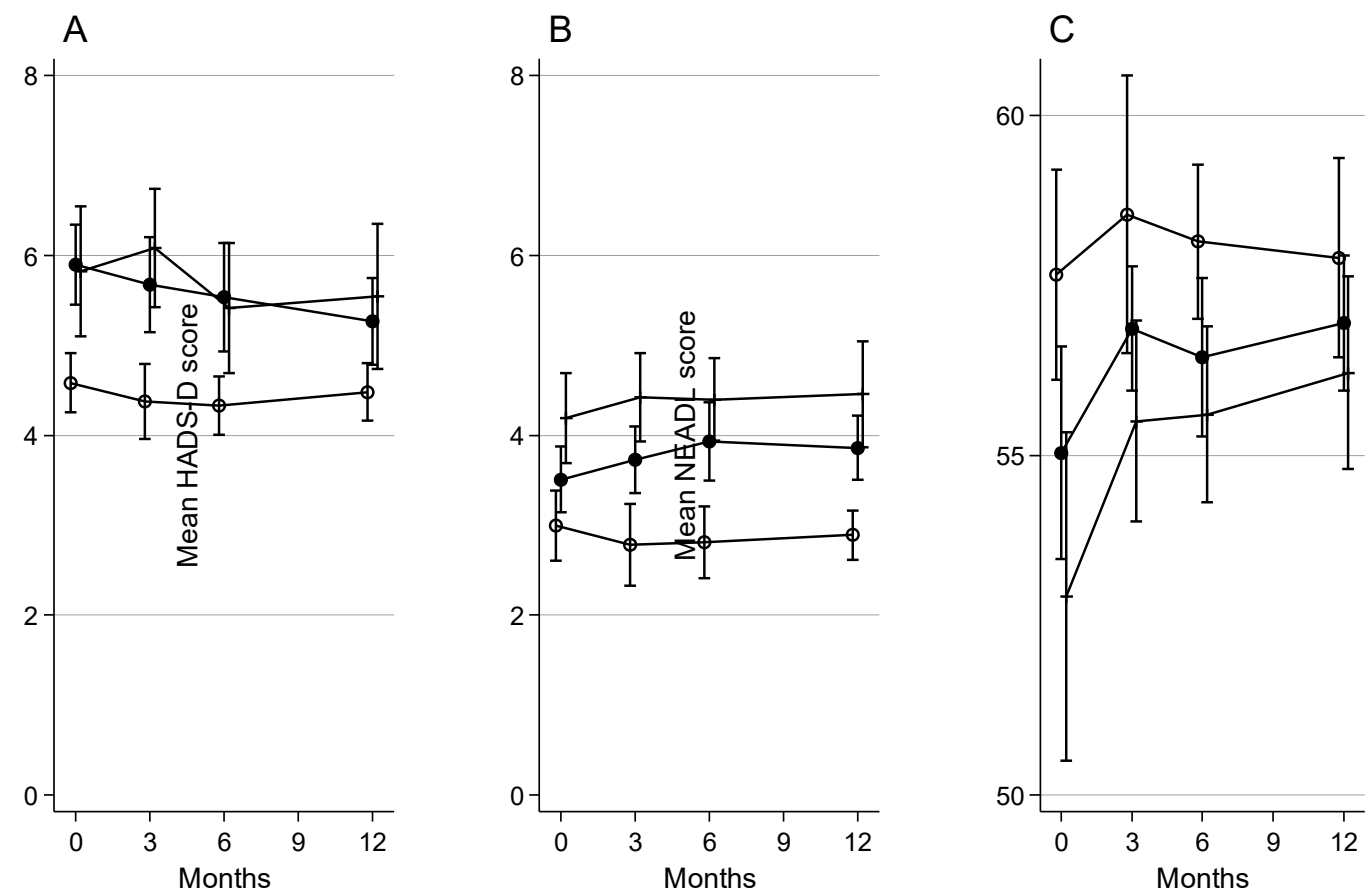
Table 1. Demographic, clinical characteristics and baseline outcome measures of FACE-

TIA participants stratified by study diagnosis group.

\begin{tabular}{|c|c|c|c|c|c|}
\hline & Control & TIA & Stroke & $\begin{array}{l}\text { Possible } \\
\text { TIA }\end{array}$ & TIA Mimic \\
\hline & $\mathrm{N}=232$ & $\mathrm{~N}=364$ & $\mathrm{~N}=183$ & $\mathrm{~N}=297$ & $\mathrm{~N}=211$ \\
\hline Age (mean, sd) & $69.9(9.7)$ & 69.8 (11.8) & 67.7 (13.1) & 66.4 (13) & $62(14.7)$ \\
\hline $\begin{array}{l}\text { Index of Multiple } \\
\text { Deprivation (mean, sd) }\end{array}$ & $20.5(12.9)$ & $17.7(12.4)$ & $22.3(15.8)$ & $18.9(13.2)$ & $19.6(13.6)$ \\
\hline Female $(\mathrm{N}, \%)$ & $98(42.2)$ & $151(41.5)$ & $84(46.2)$ & $148(50)$ & $110(52.1)$ \\
\hline $\begin{array}{l}\text { Degree level education } \\
(\mathrm{N}, \%)\end{array}$ & $54(23.3)$ & $52(14.3)$ & $15(8.2)$ & $46(15.5)$ & $38(18)$ \\
\hline $\begin{array}{l}\text { Secondary level education } \\
\text { only }\end{array}$ & $80(34.5)$ & $123(33.8)$ & $69(37.7)$ & $120(40.4)$ & $85(40.3)$ \\
\hline No formal qualification & $55(23.7)$ & $124(34.1)$ & $55(30.1)$ & $68(22.9)$ & $58(27.5)$ \\
\hline $\begin{array}{l}\text { Other qualification } \\
\text { (unknown level) }\end{array}$ & $43(18.5)$ & 65 (17.9) & $44(24.0)$ & $63(21.2)$ & $30(14.2)$ \\
\hline $\begin{array}{l}\text { Number of reported chronic } \\
\text { diseases* (mean, sd) }\end{array}$ & $1.83(1.77)$ & $1.85(1.85)$ & $1.73(1.82)$ & $1.75(1.79)$ & $1.84(1.88)$ \\
\hline Smoker (N,\%) & $11(4.8)$ & $55(15.6)$ & $30(16.9)$ & $27(9.5)$ & $30(14.4)$ \\
\hline $\begin{array}{l}\text { Days walking per week } \\
\text { (mean, sd) }\end{array}$ & $5.2(2.1)$ & $4.4(2.7)$ & $4.2(2.8)$ & $4.4(2.6)$ & $5(2.5)$ \\
\hline $\begin{array}{l}\text { Days moderate activity per } \\
\text { week (mean, sd) }\end{array}$ & $2.5(2.5)$ & $1.3(2.1)$ & $1.4(2.2)$ & $1.6(2.2)$ & $1.9(2.3)$ \\
\hline $\begin{array}{l}\text { Days vigorous activity per } \\
\text { week (mean, sd) }\end{array}$ & $1.5(2.0)$ & $0.7(1.7)$ & $0.7(1.5)$ & $0.8(1.6)$ & $1(1.8)$ \\
\hline HADS-A (mean, sd) & $4.2(3.7)$ & $5.7(4.5)$ & $5.8(4.6)$ & $6.4(4.6)$ & $6.3(4.4)$ \\
\hline Case level anxiety $(\mathrm{N}, \%)$ & $14(6.2)$ & 48 (13.9) & $27(15.3)$ & $56(20)$ & $35(17)$ \\
\hline HADS-D (mean, sd) & $2.8(2.9)$ & $3.5(3.3)$ & $4.2(3.4)$ & $4(3.5)$ & $3.4(3.2)$ \\
\hline Case level depression (N, \%) & $3(1.3)$ & $15(4.3)$ & $12(6.8)$ & $16(5.7)$ & $7(3.4)$ \\
\hline Full NEADL (mean, sd) & $57.5(11.3)$ & $54.2(11.1)$ & $52.7(12.1)$ & $54.6(12.5)$ & $56.5(10.6)$ \\
\hline NEADL Mobility (mean, sd) & $15.5(3.5)$ & $14.7(3.9)$ & $14.4(4)$ & $14.9(4.0)$ & $15.3(3.6)$ \\
\hline NEADL Kitchen (mean, sd) & $14.3(2.4)$ & $14.2(2.3)$ & $14.1(2.4)$ & $14.1(2.4)$ & $14.3(2.0)$ \\
\hline NEADL Domestic (mean, sd) & $15.4(4.1)$ & $14.4(4.4)$ & $14.2(4.7)$ & $14.6(4.6)$ & $14.9(4.2)$ \\
\hline NEADL Leisure (mean, sd) & $12.3(3.4)$ & 10.9 (3.9) & $10(3.9)$ & $11(4.0)$ & $12(3.5)$ \\
\hline
\end{tabular}

* Number of previously diagnosed conditions from arthritis, osteoporosis, asthma, COPD,

Angina, heart disease, any neurological disease, peripheral vascular disease, diabetes (type 1 or type 2), upper gastrointestinal disease, previously diagnosed depression, diagnosed anxiety, visual impairment, hearing impairment, degenerative disc disease, and obesity. 
FACE TIA

Table 2

Difference in HADS-A (anxiety), HADS-D (depression) and NEADL (disability) scores for each clinic group compared to controls, at baseline and 12 months. Estimates are derived from linear mixed models, attrition weighted and adjusted for covariates at baseline.

Estimates are shown with standard errors. Stars correspond to statistical significance at $\mathrm{p}<0.05$, and at $\mathrm{p}<0.0042$, with $\mathrm{p}<0.0042$ the critical $\mathrm{p}$-value that controls the study wide false discovery rate at $\mathrm{q} 0.05$.

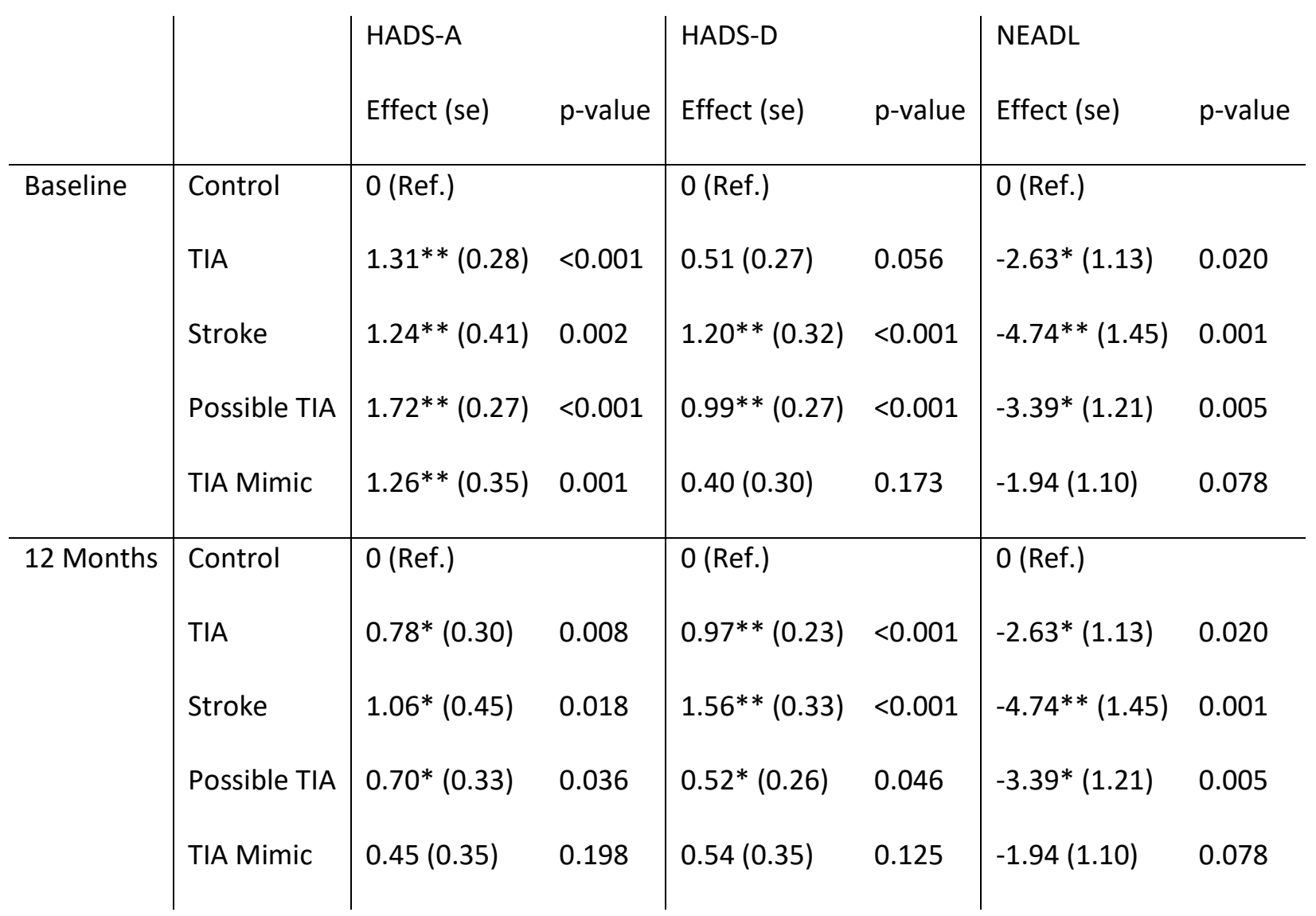

$* \mathrm{p}<0.05 * * \mathrm{p}<0.0042$ 
FACE TIA

Table 3: Change in anxiety, depression and disability between baseline and 12 month followup. Change is estimated using an attrition weighted mixed model and is estimated independently for each outcome. Estimates are shown with standard errors and p-values. Stars correspond to statistical significance at $p<0.05$ and at $p<0.0042$, with $p<0.0042$ the critical threshold required for a study wide false discovery rate of $\mathrm{q}=0.05$.

\begin{tabular}{l|ll|ll|ll} 
& HADS-A & & HADS-D & & NEADL \\
& Change (s.e.) & $p$ & Change (s.e.) & $p$ & Change (s.e.) & $p$ \\
\hline Control & $-0.10(0.12)$ & 0.417 & $-0.10(0.10)$ & 0.335 & $0.25(0.93)$ & 0.789 \\
TIA & $-0.63^{* *}(0.21)$ & 0.003 & $0.36(0.19)$ & 0.065 & $1.91^{*}(0.80)$ & 0.017 \\
Stroke & $-0.28(0.16)$ & 0.070 & $0.26(0.27)$ & 0.326 & $3.29 * *(0.90)$ & $<0.001$ \\
Possible TIA & $-1.11^{* *}(0.28)$ & $<0.001$ & $-0.57^{*}(0.23)$ & 0.011 & $1.36(0.83)$ & 0.101 \\
TIA mimic & $-0.91^{* *}(0.27)$ & 0.001 & $0.03(0.31)$ & 0.921 & $-0.35(1.09)$ & 0.749
\end{tabular}

$* \mathrm{p}<0.05 ; * * \mathrm{p}<0.0042$ 